\title{
BILATERAL DMPFC-RTMS LEADS TO SUSTAINED REMISSION IN GERIATRIC TREATMENT-RESISTANT DEPRESSION: A CASE REPORT
}

\author{
David Sender ${ }^{1}$, Bruno Palazzo Nazar ${ }^{2,3}$, Tathiana Baczynski ${ }^{1}$, Flávia Paes ${ }^{1}$, Peter Fettes ${ }^{4}$, \\ Jonathan Downar ${ }^{4,5}$, Carlos Campos ${ }^{1,6}$, Antonio Egidio Nardi ${ }^{1}$ \& Sergio Machado ${ }^{1,7}$ \\ ${ }^{1}$ Laboratory of Panic \& Respiration (LABPR), Institute of Psychiatry (IPUB), Federal University of Rio de Janeiro \\ (UFRJ), Rio de Janeiro, Brazil \\ ${ }^{2}$ Psychological Medicine, Institute of Psychiatry - King's College London - London, United Kingdom \\ ${ }^{3}$ Institute of Psychiatry (IPUB), Federal University of Rio de Janeiro (UFRJ), Rio de Janeiro, Brazil \\ ${ }^{4}$ Institute of Medical Science, University of Toronto, Canada \\ ${ }^{5}$ MRI-Guided rTMS Clinic, University Health Network, Toronto, Canada \\ ${ }^{6}$ Polytechnic Institute of Porto, Health School, Porto, Portugal \\ ${ }^{7}$ Physical Activity Neuroscience, Physical Activity Sciences Postgraduate Program - Salgado de Oliveira University, \\ Niterói, Brazil
}

received: 20.9.2016;

revised: 16.3.2017;

accepted: 1.4.2017

\section{INTRODUCTION}

Major Depressive Disorder (MDD) is a highly prevalent condition that has been labeled the current leading cause of disability adjusted life years (Ferrari et al. 2013). Approximately $30 \%$ of MDD patients are treatment resistant, not achieving remission after two or more antidepressant trials (Baldessarini et al. 2015). In the geriatric population, MDD is associated with both lower rates of remission and increased levels of recurrence (Kiosses \& Alexopoulos 2013). Geriatric MDD not only shows an impoverished response to pharmacological approaches, but also presents new treatment challenges associated with clinical limitations such as frequent metabolic issues, comorbidity and poly-medication. Hence, additional therapeutic alternatives such as repetitive transcranial magnetic stimulation (rTMS) might be beneficial in these resistant cases (Downar \& Daskalakis 2013).

Currently, the left dorsolateral prefrontal cortex (DLPFC) is the stimulation target recommended by the Food and Drug Administration when treating MDD (Lefaucheur et al. 2014). However, evidence from patients with brain lesions, functional neuroimaging, and neuromodulation studies, suggests that there are other potential rTMS targets to promote MDD remission, such as the dorsomedial prefrontal cortex (Downar \& Daskalakis 2013, Lefaucheur et al. 2014). This case report aims to describe the clinical remission experienced by an elderly patient with treatment resistant MDD after a 20 session course of bilateral $10 \mathrm{~Hz}$ DMPFC-rTMS.

\section{CASE REPORT}

The patient described in this case report provided written informed consent to engage in this study. Furthermore, the study was approved by the ethics committee of the Institute of Psychiatry - Federal University of Rio de Janeiro (protocol CAEE 2319813.0.0000.526). The patient is a 60 year-old, retired Caucasian male, referred to the Institute of Psychiatry (IPUB-UFRJ), and who presented a current severe episode of MDD without psychotic symptoms by DSM- 5 criteria. The patient had a 38-year long history of treatment-resistant MDD, during which he underwent several pharmacological interventions and psychotherapy, always with unsustained partial responses. Depressive symptoms had been escalating for the last 3 years without response to multiple courses of antidepressants (fluoxetine $60 \mathrm{mg} /$ day, paroxetine $60 \mathrm{mg} /$ day, nortriptyline $50 \mathrm{mg} /$ day, bupropion $300 \mathrm{mg} /$ day) with posteriorly adjuvant treatment (olanzapine $10 \mathrm{mg}$ /day, lithium $900 \mathrm{mg} /$ day, Haldol $5 \mathrm{mg} /$ day and valproate $1000 \mathrm{mg} /$ day).

During examination, he maintained a curved posture and complained of fatigue, anhedonia and sadness. He recurrently spoke about anxiety, feelings of despair, ruminative thoughts about dying, and feelings of severe guilt associated with life occurrences. In addition, he reported having changes in appetite and difficulties with sleep regulation, associated with a complete lack of motivation to perform daily activities or self-care. The patient had no signs or symptoms suggestive of retrospective mania, dysthymic or personality disorder. Mini Mental State Examination (MMSE) scored 27/30, and executive function clinical tests (clock test and verbal fluency test) showed no signs of cognitive impairment. The patients' most frequent complaint was a "feeling of mental laziness", impaired reasoning, and difficulties recalling names, dates and autobiographical events. Neurological and laboratory investigation showed no abnormalities.

After explaining available treatment options to the patient, rTMS was chosen. The patient completed a clinical scale to measure depression severity (Hamilton Depression Rating Scale - HAMD-17) and a self-report 
questionnaire to assess anxiety symptoms (State-Trait Anxiety Inventory - STAI-T/S) at baseline, after intervention (4-week) and at follow-up (5-months).

The patient was instructed to maintain his current medication scheme (clomipramine $150 \mathrm{mg} /$ day, mirtazapine $45 \mathrm{mg} /$ day, quetiapine $50 \mathrm{mg} /$ day, lorazepam $3 \mathrm{mg} /$ day) during the intervention. The rTMS protocol administered consisted of $10 \mathrm{~Hz}$ stimulation at $120 \%$ of the resting motor threshold for $30 \mathrm{~min}$ (2880 pulses per session), 5 times per week consecutively for 4 weeks, over the bilateral DMPFC (Fz electrode site in the 10/20 International EEG system), without neuronavigation, using the fluid-cooled AFEC-02-100 figure 8 coil with Neuro-MS/D device (Neurosoft, Brazil). Baseline, 4week and 5-month follow-up scores are presented at Figure 1.

(A)

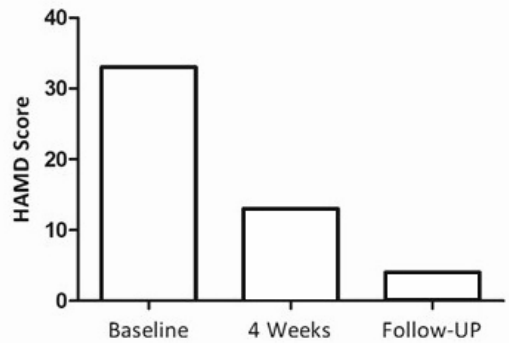

(B)

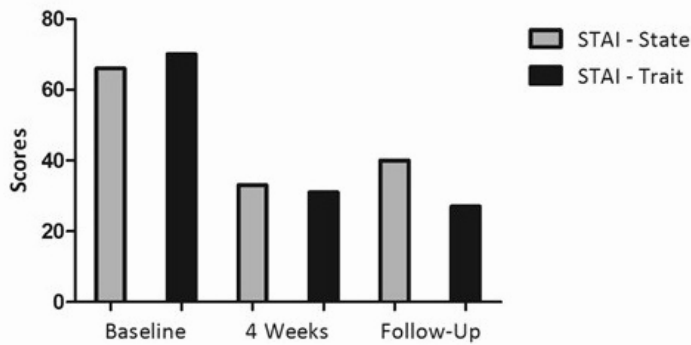

Figure 1. Scores from baseline, 4 weeks and follow-up for (A) HAMD-17 and (B) STAI

At baseline the patient had very severe depression (HAMD-17 =33) and severe trait and state anxiety (STAI-T/S $=66 / 70)$. After 4 weeks, the patient effectively responded to the treatment $(\geq 50 \%$ depressive symptoms reduction), although he still presented some mild depressive symptoms (HAMD-17 =13) and anxiety (STAI-T/S =33/31). The patient also reported a marked improvement in his memory, attention and executive function, although these findings were not confirmed with formal neuropsychological testing. Local discomfort and headache were the only reported side effects after rTMS and were managed with paracetamol. After 5 months the patient displayed full and sustained remission (HAMD-17 $=4$ ), moderate trait and mild state anxiety (STAI-T/S =40/27). As of 18 months since finishing the procedure, he has not displayed any MDD recurrence.

\section{CONCLUSIONS}

To our knowledge this is the first case of treatment resistant geriatric MDD in the literature which showed full remission after a course of bilateral DMPFC-rTMS. There are two important topics highlighted in this case report. First, our findings suggest that when it comes to the geriatric population, which commonly has several contraindications for specific pharmacological agents (Alexopoulos et al. 1996), rTMS may represent a therapeutic alternative to increase the number of treatment responders while maintaining safety and tolerability. Previous evidence from 7912 DMPFC-rTMS runs found no seizures or other serious adverse effects after stimulation suggesting its safety and tolerance for medication-resistant major depressive disorder (Bakker et al. 2015).

A recent trial also analyzed the cognitive safety profile of DMPFC-rTMS on treatment resistant depressive patients and did not found any performance hindering on learning, attention, speed of processing and cognitive flexibility measures (Schulze et al. 2016). Conversely, there were actually significant improvements with large effects on cognitive flexibility measures. In our case report there were no significant adverse effects of DMPFC-rTMS other than local discomfort and headache, with the patients actually reporting subjective improvement in cognitive performance.

Second, this case allows us to debate if DMPFCrTMS produces sustained and improved outcomes when compared to DLPFC-rTMS in cases of geriatric MDD. Most rTMS studies in MDD use the DLPFC as the preferred stimulation target for the treatment and maintenance phase (Lefaucheur et al. 2014, Drumond Marra et al. 2015, Carpenter et al. 2012, Sabesan et al. 2015). However, resting-state functional MRI evidence has highlighted the DMPFC as a "dorsal nexus" in depression, suggesting its role as a distinctive brain region that promotes increased connectivity between the cortical networks for cognitive control, affect regulation and selfreflection (Sheline et al. 2010). Furthermore, a recent retrospective case series with 185 adult patients with medication-resistant MDD analyzed the effectiveness of $10 \mathrm{~Hz}$ and theta burst DMPFC-rTMS stimulation protocols (Bakker et al. 2015). Combined data from both procedures showed similar effects in comparison to DLPFCrTMS on overall response and remission rates in clinician-rated and self-reported measures. There is also preliminary evidence showing how can resting-state functional connectivity predict treatment response to DMPFCrTMS, with higher cortical-cortical connectivity and lower cortico-thalamic, cortico-striatal, and cortico-limbic connectivity seeming to be associated to better treatment outcomes (Salomons et al. 2014, Downar et al. 2014).

Thereby, excitatory rTMS may provide enhanced emotional self-regulation and impulse control, leading to depressive symptoms amelioration. Although just emerging as a treatment for geriatric MDD, this case suggests that rTMS of the DMPFC is worth further investigation as a therapeutic intervention. Coil placement is simpler at 
the DMPFC and can be accurately achieved even without MRI guidance, making it a strong candidate for widespread use in MDD management (Downar \& Daskalakis 2013). Although our case-report showed sustained remission for 18 months without additional stimulation sessions, future studies should explore whether and when geriatric patients should receive additional DMPFCrTMS sessions during the maintenance phase. Randomized sham-controlled trials to assess the efficacy of DMPFC-rTMS in geriatric MDD are also welcomed and they could drastically improve our ability to treat these patients safely and effectively.

\section{Acknowledgements: None.}

Conflict of interest: None to declare.

\section{Contribution of individual authors:}

David Sender, Bruno Palazzo Nazar and Sergio Machado: designed the study

David Sender, Carlos Campos and Sergio Machado: did literature searches and analyses;

David Sender and Sergio Machado: did the statistical analysis;

David Sender, Bruno Palazzo Nazar, Carlos Campos and Sergio Machado: interpreted the data;

Tathiana Baczynski, Flávia Paes, Peter Fettes, Jonathan Downar, Antonio Egidio Nardi and Sergio Machado: reviewed the and approved the final version of the manuscript.

\section{References}

1. Alexopoulos GS, Meyers BS, Young RC, Kakuma T, Feder M, Einhorn A, et al.: Recovery in geriatric depression. Arch Gen Psychiatry 1996; 53:305-12. PubMed PMID: 8634008.

2. Bakker N, Shahab S, Giacobbe P, Blumberger DM, Daskalakis ZJ, Kennedy SH, et al.: rTMS of the dorsomedial prefrontal cortex for major depression: safety, tolerability, effectiveness, and outcome predictors for $10 \mathrm{~Hz}$ versus intermittent theta-burst stimulation. Brain Stimul 2015; 8:208-15. doi: 10.1016/j.brs.2014.11.002. PubMed PMID: 25465290.

3. Baldessarini RJ, Lau WK, Sim J, Sum MY, Sim K: Duration of initial antidepressant treatment and subsequent relapse of major depression. J Clin Psychopharmacol 2015; 35:75-6. doi: 10.1097/JCP.0000000000000263. PubMed PMID: 25502491.

4. Carpenter LL, Janicak PG, Aaronson ST, Boyadjis T, Brock DG, Cook IA, et al.: Transcranial magnetic stimulation (TMS) for major depression: a multisite, naturalistic, observational study of acute treatment outcomes in clinical practice. Depress Anxiety 2012; 29:587-96. doi: 10.1002/da.21969. PubMed PMID: 22689344.
5. Downar J \& Daskalakis ZJ: New targets for rTMS in depression: a review of convergent evidence. Brain Stimul 2013; 6:231-40. doi: 10.1016/j.brs.2012.08.006. PubMed PMID: 22975030.

6. Downar J, Geraci J, Salomons TV, Dunlop K, Wheeler S, McAndrews MP, et al.: Anhedonia and reward-circuit connectivity distinguish nonresponders from responders to dorsomedial prefrontal repetitive transcranial magnetic stimulation in major depression. Biol Psychiatry 2014; 76:176-85. doi: 10.1016/j.biopsych.2013.10.026. PubMed PMID: 24388670.

7. Drumond Marra HL, Myczkowski ML, Maia Memória C, Arnaut D, Leite Ribeiro P, Sardinha Mansur CG, et al.: Transcranial Magnetic Stimulation to Address Mild Cognitive Impairment in the Elderly: A Randomized Controlled Study. Behav Neurol 2015; 2015:287843. doi: 10.1155/2015/287843. PubMed PMID: 26160997; PubMed Central PMCID: PMCPMC4487699.

8. Ferrari AJ, Charlson FJ, Norman RE, Patten SB, Freedman $G$, Murray CJ, et al.: Burden of depressive disorders by country, sex, age, and year: findings from the global burden of disease study 2010. PLoS Med 2013; 10:e1001547. doi: 10.1371/journal.pmed.1001547. PubMed PMID: 24223526; PubMed Central PMCID: PMCPMC3818162.

9. Kiosses DN \& Alexopoulos GS: The prognostic significance of subsyndromal symptoms emerging after remission of latelife depression. Psychol Med 2013; 43:341-50. doi: 10.1017/S0033291712000967. PubMed PMID: 22607988; PubMed Central PMCID: PMCPMC3571621.

10. Lefaucheur JP, André-Obadia N, Antal A, Ayache SS, Baeken $C$, Benninger DH, et al.: Evidence-based guidelines on the therapeutic use of repetitive transcranial magnetic stimulation (rTMS). Clin Neurophysiol 2014; 125:2150-206. doi: 10.1016/j.clinph.2014.05.021. PubMed PMID: 25034472.

11. Sabesan P, Lankappa S, Khalifa N, Krishnan V, Gandhi R, Palaniyappan L: Transcranial magnetic stimulation for geriatric depression: Promises and pitfalls. World $J$ Psychiatry 2015; 5:170-81. doi: 10.5498/wjp.v5.i2.170. PubMed PMID: 26110119; PubMed Central PMCID: PMCPMC4473489.

12. Salomons TV, Dunlop K, Kennedy SH, Flint A, Geraci J, Giacobbe P, et al.: Resting-state cortico-thalamic-striatal connectivity predicts response to dorsomedial prefrontal rTMS in major depressive disorder. Neuropsychopharmacology 2014; 39:488-98. doi: 10.1038/npp.2013.222. PubMed PMID: 24150516; PubMed Central PMCID: PMCPMC3870791.

13. Schulze L, Wheeler S, McAndrews MP, Solomon CJ, Giacobbe P, Downar J: Cognitive safety of dorsomedial prefrontal repetitive transcranial magnetic stimulation in major depression. Eur Neuropsychopharmacol, 2016. doi: 10.1016/j. euroneuro.2016.04.004. PubMed PMID: 27157074.

14. Sheline YI, Price JL, Yan Z, Mintun MA: Resting-state functional MRI in depression unmasks increased connectivity between networks via the dorsal nexus. Proc Natl Acad Sci USA 2010; 107:11020-5. doi: 10.1073/pnas.1000446107. PubMed PMID: 20534464; PubMed Central PMCID: PMCPMC2890754.

Correspondence:

Sergio Machado, MD, PhD

Laboratory of Panic $\mathcal{E}$ Respiration, Institute of Psychiatry

Federal University of Rio de Janeiro

Rio de Janeiro, Brazil

E-mail:secm80@gmail.com 